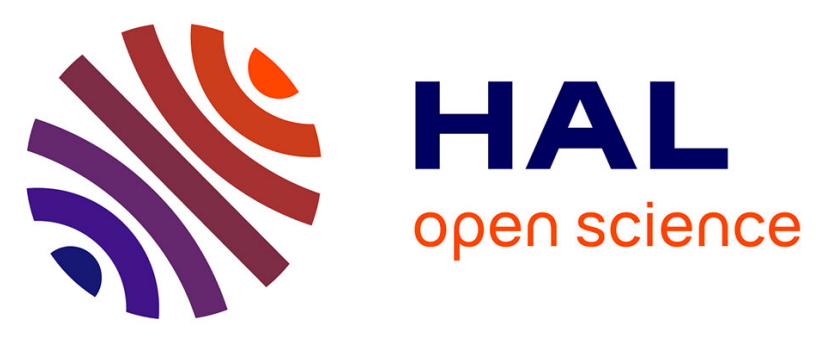

\title{
Burnout symptomatology and social support at work independent of the private sphere: a population-based study of French teachers
}

\author{
Sofia Temam, Nathalie Billaudeau, Marie-Noël Vercambre
}

\section{- To cite this version:}

Sofia Temam, Nathalie Billaudeau, Marie-Noël Vercambre. Burnout symptomatology and social support at work independent of the private sphere: a population-based study of French teachers. International Archives of Occupational and Environmental Health, 2019, 92 (6), pp.891-900. 10.1007/s00420019-01431-6 . hal-02918469

\author{
HAL Id: hal-02918469 \\ https://hal.science/hal-02918469
}

Submitted on 20 Aug 2020

HAL is a multi-disciplinary open access archive for the deposit and dissemination of scientific research documents, whether they are published or not. The documents may come from teaching and research institutions in France or abroad, or from public or private research centers.
L'archive ouverte pluridisciplinaire HAL, est destinée au dépôt et à la diffusion de documents scientifiques de niveau recherche, publiés ou non, émanant des établissements d'enseignement et de recherche français ou étrangers, des laboratoires publics ou privés. 
Burnout symptomatology and social support at work independent of the private sphere: a population-based study of French teachers

Sofia Temam a, Nathalie Billaudeau ${ }^{a}$, Marie-Noël Vercambre a

a MGEN Foundation for Public Health, Paris, France

Sofia Temam - MGEN Foundation for Public Health, Paris, France - stemam@mgen.fr

Nathalie Billaudeau - MGEN Foundation for Public Health, Paris, France - nbillaudeau@mgen.fr

Marie-Noël Vercambre - MGEN Foundation for Public Health, Paris, France - mvercambre@mgen.fr

\section{Corresponding author:}

Sofia Temam

MGEN Foundation for Public Health

3 square Max Hymans

75748 Paris Cedex 15

stemam@mgen.fr

Phone number: +33140472764

\section{Acknowledgements:}

The authors would like to thank Fabien Gilbert and Pascale Lapie-Legouis, as well as the Office of Statistical Studies on Personnel of the French Ministry of Education (Direction de l'évaluation, de la prospective et de la performance - DEPP).

\section{Declaration of Interest statement:}

The authors declare that they have no competing interests. 


\section{ABSTRACT}

Purpose: To investigate the relationship between social support at work and burnout among teachers, independent of the teachers' private social and environmental context.

Methods: In the 2013 “Teachers' Quality of Life" population-based study (France, $n=2,653$ ), burnout symptomatology was assessed using the Maslach Burnout Inventory. The score for each dimension (emotional exhaustion, depersonalization and personal accomplishment) was dichotomized using extreme tertiles. Global score for social support at work and subscores by source and type were derived from the Karasek Job Content Questionnaire and were categorized into tertiles (low, medium, and high). The private context was appraised through the "social relationships" and "environment" subscales of the short version of the World Health Organization Quality of Life questionnaire (WHOQOL-BREF). Associations between social support at work and burnout indicators were evaluated among 2,473 teachers with complete data, using logistic regression models adjusted for the private context plus sociodemographic and work-related characteristics.

Results: Eight percent of the teachers showed simultaneously high emotional exhaustion, high depersonalization and low personal accomplishment symptoms and were considered as burnout cases. After controlling for the private context, teachers who reported high social support at work were significantly less likely to report burnout (odds ratio [95\% confidence interval] high vs. low=0.62 [0.40, 0.98]). When distinguishing the source of social support at work, only high social support from supervisors remained significantly associated with a lower risk of burnout $(0.43[0.27,0.71])$.

Conclusions: Improving social support at work, especially from a hierarchical point of view, may be an effective target to promote teacher's wellbeing.

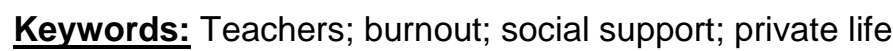




\section{INTRODUCTION}

A number of studies have reported a positive relationship between social support and both physical and mental health all along the life course (Holt-Lunstad et al. 2010; Gariepy et al. 2016). Social support is a multidimensional concept that refers to helpful social interactions that people perceive to be available or that are actually provided. Social support can fulfill different functions (i.e., instrumental, financial, informational, or emotional) and be provided by several sources in the private life (i.e., family or friends) or in the work environment (i.e., supervisors or coworkers) (Berkman et al. 2000). In the literature, different physiologic pathways and psychologic mechanisms have been proposed to explain the positive effect of social support on health (Uchino 2006), in particular, the main effect model and the stress-buffering model. According to the main effect model, social support is beneficial for well-being independently of level of stress to which individuals are exposed, for example by promoting healthy behaviors or facilitate access to resources and material goods. According to the stress-buffering model, social support may provide resources that would moderate the deleterious effect of stressful events on health (Berkman et al. 2000; Holt-Lunstad et al. 2010). These different mechanisms linking social support to health are still debated (Uchino et al. 2012). As a modifiable health-related factor, social support is increasingly suggested as a target for interventions in chronic diseases (Pikhart and Jitka Pikhartova 2015).

In the occupational health context, several studies have suggested a beneficial effect of social support in the workplace to help workers to cope with stressful situations (Uchino et al. 2012). As such, prevention programs designed to enhance social support at work may represent an effective strategy for promoting wellbeing at work (Naghieh et al. 2015) and to reduce mental illness, which is considered today as the leading cause of sickness absence and long term work incapacity in most high-income countries (Harvey et al. 2017). However, most studies did not account for social support outside of the workplace which may also play a role in assisting workers to cope with job-related stress (Hakanen et al. 2008; Beauregard et al. 2011; Hakanen and Bakker 2017) but is not accessible by organizational-level interventions. Furthermore, other aspects of life domains, including the perception of one's environment, has been related to mental well-being (Guite et al. 2006) and also to work outcomes such as productivity, absenteeism or turnover (Sears et al. 2013). That is why it is important to develop an integrative approach by including both occupational and non-occupational determinants 
of workers' mental health (Beauregard et al. 2011; Bianchi et al. 2014; Bianchi 2016; Hakanen and Bakker 2017). From a methodological point of view, not taking into account the interplay between work-related and non-work-related factors may bias the estimations of the specific impact of social support at work (Madsen et al. 2014).

In this context, the present analysis is focused on teachers, who have been identified as particularly at risk of stress-related disorders (Wieclaw et al. 2005; Kokkinos 2007) including the burnout syndrome (Vercambre et al. 2009; Schonfeld 2017). The burnout syndrome is commonly defined as a psychological syndrome resulting of a prolonged exposure to work-related stressors and consisting of emotional exhaustion, depersonalization and a sense of reduced personal accomplishment at work (Maslach and Leiter 2016). Among the many studies of risk factors for burnout among teachers (Hakanen et al. 2006; Kokkinos 2007; Vercambre et al. 2009), some have included social support as an exposure of interest (Russell et al. 1987; Burke and Greenglass 1993; Greenglass et al. 1994; Fiorilli et al. 2015; Arvidsson et al. 2016). However, only a few took concomitantly into account nonwork risk factors for burnout and they reported inconsistent results (Russell et al. 1987; Burke and Greenglass 1993; Greenglass et al. 1994). Preventing burnout among teachers, who represent a nonnegligible part of the active population in developed countries (approximately $4 \%$ in France), is an important issue, as such approach may have favorable consequences not only for teachers' health but also for pupils' well-being (Oberle and Schonert-Reichl 2016), the school organization and efficiency of the education system in general (Naghieh et al. 2015).

Taking advantages of the detailed work and non-work information available on teachers from the French population-based study "Qualité de vie des enseignants" ("Teachers' Quality of Life"), we tested the hypothesis that higher social support at work was associated with a lower probability of burnout among teachers, independent of their private social and environmental context. The first aim was to investigate the relationship between social support at work and burnout, considering the teachers' private life context. A secondary aim was to explore the association between specific source (i.e., supervisors and coworkers) and type (i.e., emotional and instrumental) of social support at work and burnout. 


\section{MATERIALS AND METHODS}

\section{Population}

The population-based postal study "Qualité de vie des enseignants" ("Teachers' Quality of Life") is a cross-sectional study that was carried out in 2013 by the MGEN Foundation for Public Health (www.fondationmgen.fr), with the technical support of the statistical department ("Direction de l'évaluation, de la prospective et de la performance - DEPP") of the French Ministry of Education (Zavidovique et al. 2017). A detailed questionnaire was sent to the workplace of 5,000 in-service teachers of all grades (from nursery school to university) who were randomly selected among the teaching staff administrative list of the French Ministry of Education after stratification by sex, age and type of school (public, public in a priority education area or private school under contract). A satisfactorily completed questionnaire was returned by 2,653 participants ( $53 \%$ response rate). Along with sociodemographic information, detailed work-related characteristics were recovered, and several standardized instruments were used to measure quality of life and well-being at work and in general. In addition to the postal questionnaire, a set of administrative data such as teachers' professional corps and school characteristics were collected directly from the Ministry of education databases. This study was approved by both of the national authorities then responsible for protecting privacy and personal data: the "Comité Consultatif sur le Traitement de l'Information en matière de Recherche dans le domaine de la Santé" (CCTIRS) and the "Commission Nationale de I'Informatique et des Libertés" (CNIL).

\section{Burnout symptomatology}

Burnout symptomatology was self-evaluated using the French version of the Maslach Burnout Inventory (MBI) (Maslach and Jackson 1981) adapted to educational settings (MBI-Educators Survey (Iwanicki and Schwab 1981)). The MBI includes 22 items evaluating three dimensions: emotional exhaustion (9 items), depersonalization (5 items) and personal accomplishment (8 items). Each item relates to a specific symptom and can be answered on a 7-point Likert scale ranging from "never" $(=0)$ to "daily" $(=6)$. The scores of the three burnout dimensions were dichotomized using extreme tertiles, as previously suggested (Brenninkmeijer and VanYperen 2003; Vercambre et al. 2009). Emotional exhaustion and depersonalization were respectively assigned a value of ' 1 ' (presence of symptom) 
when scores were above the 2nd tertile, whereas personal accomplishment was assigned a value of ' 1 ' if the score was below the 1st tertile. People with high scores for emotional exhaustion and depersonalization together with a low score for personal accomplishment were considered as burnout cases. The MBI has been widely used in the literature (Bianchi et al. 2014), and the French version has been validated in both general and educational settings (Pedrabissi et al. 1993; Dion and Tessier 1994; Fiorilli et al. 2015). In the "Teachers' Quality of Life" study, the Cronbach's alpha coefficients were 0.89 for the emotional exhaustion subscale, 0.65 for depersonalization, and 0.76 for personal accomplishment (Table S1).

\section{Social support at work}

Social support at work was assessed using the French version of the Job Content Questionnaire (JCQ) based on the demand-control-support model developed by Karasek and Theorell to measure the psychosocial work environment (Karasek and Theorell 1990). The questionnaire is composed of three scales, answered on a 4-point Likert scale: "psychological demand" (9 items), "decision latitude" (9 items) and "social support" (8 items), with Cronbach's alpha coefficients in the present study of $0.69,0.78$ and 0.82 , respectively (Table $\mathrm{S} 1$ ). The social support scale specifically includes eight items that allow the calculation of a global score for social support at work, as well as subscores by sources (i.e., supervisors and coworkers) and types (i.e., emotional and instrumental) (Table S2). The French version has been evaluated and used in various worker samples (Niedhammer 2002). In the present study, the scores for overall social support at work and social support by source and type were categorized into tertiles, to define "low", "medium" and "high" levels of social support.

\section{Private social and environmental context}

To evaluate the private context of the teacher, we used two domains of the French abbreviated version of the World Health Organization Quality of Life (WHOQOL)-BREF questionnaire (Baumann et al. 2010), namely, the "social relationships" scale (3 items: personal relationships, social support and sexual activity; score range: $3-15$ ) and the "environment" scale (8 items including financial resources, physical safety, leisure activities, home physical environment and transportation; score range: $5-40$ ). A higher score indicates a better perception of social relationships and the living environment. Both scales were standardized (z-score) and introduced in the model as continuous variables. In the 
present setting, Cronbach's alpha coefficients were 0.72 for both scales (Table S1). In addition to these two variables, some family-related indicators were available (i.e., marital status and having children aged less than 7 years old), and these indicators were introduced in a sensitivity analysis to complete the information on the private context.

\section{Further covariates}

In addition to sociodemographic characteristics (age and sex), work-related characteristics that could be linked to both social support at work and burnout were considered 1) at the "teacher-level", i.e., teaching level (nursery school, primary school, secondary school or university), psychological demand and decision latitude measured through the JCQ (Karasek and Theorell 1990), psychological violence at work according to Leymann's definition (Niedhammer et al. 2006) in three categories, i.e., neither victim nor witness, witness, or victim; and 2) at the "school-level", i.e., type of school (public, public in a priority education area, or private), size ( $\leq 199,200-999$ or $\geq 1000$ pupils) and school location (rural, suburban or urban). For each of these covariates, missing values represented less than five percent. To limit sample attrition, they were imputed with the most represented value for categorical variables and with the average score of the study population for continuous variables.

\section{Statistical analysis}

First, bivariate analyses were carried out to explore the association between the level of social support at work and potential confounding factors. Second, Pearson's correlation coefficients were computed between scores of social support at work, the private social and environmental context and the three burnout dimensions. Third, associations between the level of social support at work and burnout were tested using a logistic regression analysis with adjustment variables being entered in blocks. We estimated odds ratios adjusted for sociodemographic characteristics (Model 1), then work-related characteristics were added (Model 2), and then private social and environmental context variables were added (Model 3). Only adjustment variables with a $p$-value $<0.15$ from the bivariate analyses were entered into the models. The three burnout dimensions (high emotional exhaustion, high depersonalization and reduced personal accomplishment), as well as the burnout outcome (burnout cases), were modeled separately. Additional analyses were performed to investigate the specific effect of the source (i.e., supervisors and coworkers) and the type (i.e., emotional and instrumental) of social 
support at work on burnout. Furthermore, several sensitivity analyses were performed to test the robustness of our results. As the "social relationships" subscale of the WHOQOL-BREF questionnaire was not originally built to evaluate social support outside of the workplace, it could overlap our measure of social support at work evaluated through the JCQ (e.g., a colleague can also be considered as a friend). Therefore, to isolate the private dimension of social support, we regressed the WHOQOL-BREF "social relationships" subscale on the JCQ "social support at work" subscale. The residuals of this regression, subsequently referred to as the "private component" of the WHOQOLBREF "social relationships" subscale, were used as an alternative to the initial subscale to adjust for the private social support. Finally, we ran stratified analyses by age and by sex and linear regression models with the $\mathrm{MBI}$ dimensions treated as continuous variables. All analyses were conducted using Stata/SE (version 13; Stata Corp., College Station, TX, USA) with the "SVY" command to account for the sampling design.

\section{RESULTS}

Among the 2,653 teachers who answered the questionnaire, we excluded 180 participants (7\%) with missing data for any of the variables of interest (burnout, social support at work, private social and environment context) from the analysis. There was no significant difference in terms of age, sex or teaching level between the included and excluded teachers (not shown). Consistent with the exhaustive statistics of teaching staff at the national level, our study sample of 2,473 teachers consisted mainly of women (67\%) who were 23 to 69 years of age.

\section{Characteristics of the population by level of social support at work}

Compared to teachers who reported a low level of social support at work, teachers who reported a higher level of support were younger. Those teachers also declared better working conditions, which were characterized by a lower score for psychological demand, a higher score for decision latitude, and less exposure to psychological violence. Regarding school-related characteristics, teachers in private schools and those teaching in medium-sized schools (between 200-999 pupils) more often reported a high level of social support at work (Table 1). 


\section{Correlation between sources and types of social support and burnout}

The different sources and types of social support at work were strongly correlated with each other (Pearson's correlation coefficient: $r \geq 0.7$ ), except between social support from supervisors and from coworkers $(r=0.2)$ (Table 2). Consistent with our hypothesis, social support at work was positively correlated with the private social and environmental context, indicating that teachers who reported higher social support at work were also more satisfied in regard to their private social and their living environment; however, the correlations were relatively weak $(r<0.3)$. Additionally, scores for social support at work and the private social and environmental context were negatively correlated with emotional exhaustion and depersonalization and positively correlated with personal accomplishment.

\section{Social support at work and burnout, independent of the private social and environmental context}

Approximately $7.5 \%$ of the teachers showed simultaneously high emotional exhaustion, high depersonalization and low personal accomplishment and were considered as burnout cases. The proportion of burnout cases significantly decreased when social support increased, with a prevalence of $12.1 \%, 8.2 \%$ and $3.6 \%$ for low, medium and high levels of social support, respectively (Table 3). In the model adjusted for age and sex only (Model 1), high social support at work was associated with a lower risk of reporting burnout, with a significant linear trend. Social support at work was also significantly and negatively associated with the three burnout dimensions. When the work-related characteristics and the private context variables were added as adjustment variables (Model 3), the strength of the associations was substantially attenuated, but the social support at work remained significantly associated with less burnout, in particular with higher level of personal accomplishment (Table 3). When considering the specific source and type of social support at work and burnout (Table 4), a significant decreased risk of burnout was observed among teachers who reported high social support from their supervisors, but not among those who reported high social support from their coworkers, and among teachers who reported high emotional support but not among those who reported high instrumental support. In the sensitivity analysis, when we used the private component of the "social relationships" WHOQOL-BREF subscale as adjustment variable rather than the initial scale, the results corroborated the relationship between social support at work and burnout protection independent of the private support (Table S3). The linear regression models provided similar results 
compared to the logistic regression models (Table S4). Stratification by age and by sex did not modify the results (not shown). When we added the family-related indicators to the main model, the results were virtually unchanged (not shown).

\section{DISCUSSION}

In this representative sample of French teachers, high social support at work was associated with a lower level of burnout. After controlling for teachers' private social and environmental context, the association, although attenuated, remained significant. As supported by the separate analysis of the three burnout dimensions, the putative protective effect of social support at work may act especially through the preservation of personal accomplishment at work. The association between social support at work and burnout symptomatology differed according to the source or type considered. First, we found that teachers who reported higher social support from their supervisors had a lower risk of reporting burnout, whereas the association was not significant with social support from coworkers. Second, we observed a more consistent inverse association between emotional support and burnout symptomatology than with instrumental support. Several sensitivity analyses corroborated our results.

Burnout is usually defined as a consequence of work-related chronic stress exposure (Maslach and Leiter 2016), and few studies have considered the workers' private life context when studying the effect of social support at work on burnout (Bianchi 2016). As nonoccupational factors may confound the association but are not accessible by organizational-level interventions, it is important to take them into account in the analysis to identify effective measures to promote well-being at work (Madsen et al. 2014). To evaluate the private life context, we considered individuals' perceptions of the quality of their social relationships and their living environment. It is well recognized that social relationships have a positive impact on mental health (Holt-Lunstad et al. 2010). Likewise, some studies reported that having a positive perception of characteristics relating to neighborhood social and physical environments was related to a lower prevalence of mental health conditions (Sears et al. 2013; Van Dyck et al. 2015). In our study, the indicators of the private social and environmental context were positively but weakly correlated with social support at work, indicating that if they were linked, they also provided different information. Therefore, overadjustment bias due to collinearity is likely to be 
limited in the fully adjusted model. Overall, our data support the idea that the private context was a source of confounding in the analysis of the work context/health relationship. In particular, we observed among teachers that social support at home and at work were positively linked. Adjusting for the teachers' private context decreased the estimated effect of social support at work on burnout, but social support from both sources remained significantly associated with burnout, suggesting that both had an independent effect on health and well-being.

To study how social support at work may influence burnout symptomatology independent of confounders, we adopted a pragmatic exposure/outcome epidemiological approach based on instruments well-established in the French occupational health context, such as the JCQ and the MBI, together with more punctual items that had been recognized as important determinants of wellbeing among teachers (Kokkinos 2007). Other instruments to evaluate burnout (e.g. Oldenberg Bumout Inventory (Demerouti et al. 2003)) and various conceptual models in the field of occupational health (e.g. the Job Demands-Resources model) have more lately been developed (Maslach and Leiter 2016; Hakanen and Bakker 2017); using such alternatives would be of interest to further test the robustness of our results.

Few and rather ancient studies have investigated the association between social support and burnout among teachers along with non-occupational factors. Burke and Greenglass analyzed the effect of social support on burnout among 833 UK teachers (Burke and Greenglass 1993). In their study, they included family-related indicators (i.e., presence of children and marital status) and social support from three sources at work and outside of work. They found a significant negative association between social support and lower personal accomplishment, but not with the emotional exhaustion and depersonalization dimensions, which is consistent with our observations. Marital status and the presence of children were not associated with burnout. However, they did not provide the relative contribution of each source of social support.

Our results also suggested that the putative effect of social support on burnout depended on the source or type of social support considered. First, social support from supervisors appeared to be more determinant than social support from coworkers when coping with burnout symptomatology. This result is in line with studies that similarly explored the distinctive role of social support from supervisors and coworkers on workers' wellbeing and job satisfaction among teachers (Russell et al. 1987; 
Arvidsson et al. 2016; Yuh and Choi 2017), nurses (Constable and Russell 1986) and employees (Tang et al. 2014). Interestingly, we observed that social support from supervisors and coworkers was weakly correlated. This result supported that both sources of support reflect, at least in part, a different aspect of social support at work and thus could be analyzed separately. Second, we observed that emotional support was more consistently linked with a lower risk of burnout than was instrumental support. Emotional support involves communication of caring, empathy and esteem (Cohen 2004). Some authors argue that certain types of social support are inherently supportive, especially the emotional component; when interactions are seen as emotionally supportive, the support recipient is likely to evaluate the interactions as supportive (Cohen 2004; Gariepy et al. 2016). In addition, teaching is recognized as a profession with a high emotional demand (Kinman et al. 2011). Thus, emotional support could help teachers deal with this emotional demand more effectively than could instrumental support.

The strengths of our study include the robustness of the results in this relatively large and representative sample of French teachers, which allowed us to consider numerous potential confounding factors, including several sociodemographic and both private and work characteristics. The response rate is comparable to that of various health surveys among teachers (Hakanen et al. 2006) and quite higher than that of health surveys among less educated occupational groups (Santin et al. 2014). As compared to respondents, non-respondents in the "Teachers Quality of Life study" were more likely to be male and to teach in higher education. They were also younger. Moreover, to enrich the self-reported data from validated questionnaires such as the MBI, JCQ and WHOQOLBREF, we used comprehensive administrative data that limited the risk of missing values on several covariates (e.g., sex, age, teaching level). Some limitations should also be considered. Due to the cross-sectional design, we were not able to address causality. In particular, as people with a high level of burnout may be more prone to perceive less support or to set themselves apart, there is a possibility of reverse causality. Longitudinal data would be needed to strengthen the level of evidence and to better understand the underlying mechanism. Additionally, the questionnaire was sent to the teachers' workplace, more often excluding teachers on long-term sick leave. However, we have no reason to believe that among these teachers whose health was deteriorated, the association between social support and burnout would be different, so the impact of response bias in our association study remained limited. 
In conclusion, our results are consistent with prior research suggesting that social support from supervisors might be considered as a valuable resource to promote well-being at work among teachers. From a methodological point of view, we observed that the association between social support at work and burnout remained significant but was attenuated when controlling for the private context, highlighting the importance of an integrative approach of both the residential and work environments in epidemiologic studies. Our results must be confirmed in longitudinal studies that concomitantly investigate work and non-work determinants of workers' mental health to better inform healthy workplace research, intervention, and policy. 


\section{REFERENCES}

Arvidsson I, Håkansson C, Karlson B, et al (2016) Burnout among Swedish school teachers - a crosssectional analysis. BMC Public Health 16:823. doi: 10.1186/s12889-016-3498-7

Baumann C, Erpelding ML, Régat S, et al (2010) The WHOQOL-BREF questionnaire: French adult population norms for the physical health, psychological health and social relationship dimensions. Rev Epidemiol Sante Publique 58:33-39. doi: 10.1016/j.respe.2009.10.009

Beauregard N, Marchand A, Blanc M-E (2011) What do we know about the non-work determinants of workers' mental health? A systematic review of longitudinal studies. BMC Public Health 11:439. doi: 10.1186/1471-2458-11-439

Berkman LF, Glass T, Brissette I, Seeman TE (2000) From social integration to health: Durkheim in the new millennium. Soc Sci Med 51:843-57. doi: 10.1016/S0277-9536(00)00065-4

Bianchi R (2016) Occupational and non-occupational strains should be concomitantly considered in research on burnout, organizational commitment, and turnover intention. Int J Nurs Stud 53:403404. doi: 10.1016/j.ijnurstu.2015.10.005

Bianchi R, Truchot D, Laurent E, et al (2014) Is burnout solely job-related? A critical comment. Scand J Psychol 55:357-361. doi: 10.1111/sjop.12119

Brenninkmeijer V, VanYperen N (2003) How to conduct research on burnout: advantages and disadvantages of a unidimensional approach in burnout research. Occup Environ Med 60 Suppl 1:i16-i20. doi: 10.1136/oem.60.suppl_1.i16

Burke R, Greenglass E (1993) Work stress, role conflict, social support, and psychological burnout among teachers. Psychol Rep 371-380

Cohen S (2004) Social Relationships and Health. Am Psychol 59:676-684. doi: 10.1037/0003066X.59.8.676

Constable JF, Russell DW (1986) The Effect of Social Support and the Work Environment upon Burnout among Nurses. J Human Stress 12:20-26. doi: 10.1080/0097840X.1986.9936762

Demerouti E, Bakker AB, Vardakou I, Kantas A (2003) The convergent validity of two burnout 
instruments: A multitrait-multimethod analysis. Eur. J. Psychol. Assess.

Dion G, Tessier R (1994) Validation de la traduction de l'Inventaire d'épuisement professionnel de Maslach et Jackson. [Validation of a French translation of the Maslach Burnout Inventory (MBI).]. Can J Behav Sci Can des Sci du Comport 26:210-227. doi: 10.1037/0008-400x.26.2.210

Fiorilli C, Gabola P, Pepe A, et al (2015) The effect of teachers' emotional intensity and social support on burnout syndrome. A comparison between Italy and Switzerland. Rev Eur Psychol Appl 65:275-283. doi: 10.1080/10615800701742461

Gariepy G, Honkaniemi H, Quesnel-Vallee A (2016) Social support and protection from depression: systematic review of current findings in Western countries. Br J Psychiatry 209:284-293. doi: 10.1192/bjp.bp.115.169094

Greenglass ER, Fiksenbaum L, Burke RJ (1994) The relationship between social support and burnout over time in teachers. J Soc Behav Personal 9:219-230

Guite HF, Clark C, Ackrill G (2006) The impact of the physical and urban environment on mental wellbeing. Public Health 120:1117-1126. doi: 10.1016/j.puhe.2006.10.005

Hakanen JJ, Bakker AB (2017) Born and bred to burn out: A life-course view and reflections on job burnout. J Occup Health Psychol 22:354-364. doi: 10.1037/ocp0000053

Hakanen JJ, Bakker AB, Schaufeli WB (2006) Burnout and work engagement among teachers. J Sch Psychol 43:495-513. doi: 10.1016/j.jsp.2005.11.001

Hakanen JJ, Schaufeli WB, Ahola K (2008) The job demands-resources model: A three-year crosslagged study of burnout, depression, commitment, and work engagement. Work Stress 22:224241. doi: $10.1080 / 02678370802379432$

Harvey SB, Modini M, Joyce S, et al (2017) Can work make you mentally ill? A systematic metareview of work-related risk factors for common mental health problems. Occup Environ Med 74:301-310. doi: 10.1136/oemed-2016-104015

Holt-Lunstad J, Smith TB, Layton JB (2010) Social relationships and mortality risk: A meta-analytic review. PLoS Med 7:. doi: 10.1371/journal.pmed.1000316

Iwanicki EF, Schwab RL (1981) A Cross Validation Study of the Maslach Burnout Inventory. Educ 
Karasek R, Theorell T (1990) Healthy Work: Stress, Productivity, and the Reconstruction of Working Life, Basic Book. New York

Kinman G, Wray S, Strange C (2011) Emotional labour, burnout and job satisfaction in UK teachers: The role of workplace social support. Educ Psychol 31:843-856. doi:

$10.1080 / 01443410.2011 .608650$

Kokkinos CM (2007) Job stressors, personality and burnout in primary school teachers. Br J Educ Psychol 77:229-243. doi: 10.1348/000709905X90344

Madsen IE, Jorgensen AF, Borritz M, et al (2014) Is the association between high strain work and depressive symptoms modified by private life social support: a cohort study of 1,074 Danish employees? BMC Public Health 14:698. doi: 10.1186/1471-2458-14-698

Maslach C, Jackson SE (1981) The measurement of experienced burnout. J Organ Behav 2:99-113. doi: $10.1002 / j o b .4030020205$

Maslach C, Leiter MP (2016) Understanding the burnout experience: Recent research and its implications for psychiatry. World Psychiatry 15:103-111. doi: 10.1002/wps.20311

Naghieh A, Montgomery P, Bonell CP, et al (2015) Organisational interventions for improving wellbeing and reducing work-related stress in teachers. In: Naghieh A (ed) Cochrane Database of Systematic Reviews. John Wiley \& Sons, Ltd, Chichester, UK, pp 10-13

Niedhammer I (2002) Psychometric properties of the French version of the Karasek Job Content Questionnaire: A study of the scales of decision latitude, psychological demands, social support, and physical demands in the GAZEL cohort. Int Arch Occup Environ Health 75:129-144. doi: $10.1007 / \mathrm{s} 004200100270$

Niedhammer I, David S, Degioanni S (2006) La version française du questionnaire de Leymann sur la violence psychologique au travail : le « Leymann Inventory of Psychological Terror » (LIPT). Rev Epidemiol Sante Publique 54:245-262. doi: 10.1016/S0398-7620(06)76720-7

Oberle E, Schonert-Reichl KA (2016) Stress contagion in the classroom? The link between classroom teacher burnout and morning cortisol in elementary school students. Soc Sci Med 159:30-37. 
doi: 10.1016/j.socscimed.2016.04.031

Pedrabissi L, Rolland JP, Santinello M (1993) Stress and burnout among teachers in italy and france. J. Psychol. Interdiscip. Appl. 127:529-535

Pikhart H, Jitka Pikhartova (2015) The relationship between psychosocial risk factors and health outcomes of chronic diseases : a review of the evidence for cancer and cardiovascular diseases. $1-40$

Russell DW, Altmaier E, Van Velzen D (1987) Job-related stress, social support, and burnout among classroom teachers. J Appl Psychol 72:269-274. doi: 10.1037//0021-9010.72.2.269

Santin G, Geoffroy B, Bénézet L, et al (2014) In an occupational health surveillance study, auxiliary data from administrative health and occupational databases effectively corrected for nonresponse. J Clin Epidemiol 67:722-730. doi: 10.1016/j.jclinepi.2013.10.017

Schonfeld IS (2017) Educator Stress. doi: 10.1007/978-3-319-53053-6

Sears LE, Shi Y, Coberley CR, Pope JE (2013) Overall well-being as a predictor of health care, productivity, and retention outcomes in a large employer. Popul Health Manag 16:397-405. doi: 10.1089/pop.2012.0114

Tang S, Siu O, Cheung F (2014) A Study of Work-Family Enrichment among Chinese Employees: The Mediating Role between Work Support and Job Satisfaction. Appl Psychol 63:130-150. doi: 10.1111/j.1464-0597.2012.00519.x

Uchino BN (2006) Social Support and Health: A Review of Physiological Processes Potentially Underlying Links to Disease Outcomes. J Behav Med 29:377-387. doi: 10.1007/s10865-0069056-5

Uchino BN, Bowen K, Carlisle M, Birmingham W (2012) Psychological pathways linking social support to health outcomes: A visit with the "ghosts" of research past, present, and future. Soc Sci Med 74:949-957. doi: 10.1016/j.socscimed.2011.11.023

Van Dyck D, Teychenne M, McNaughton SA, et al (2015) Relationship of the perceived social and physical environment with mental health-related quality of life in middle-aged and older adults: Mediating effects of physical activity. PLoS One 10:1-16. doi: 10.1371/journal.pone.0120475 
Vercambre M-N, Brosselin P, Gilbert F, et al (2009) Individual and contextual covariates of burnout: a cross-sectional nationwide study of French teachers. BMC Public Health 9:333. doi:

$10.1186 / 1471-2458-9-333$

Wieclaw J, Agerbo E, Mortensen PB, Bonde JP (2005) Occupational risk of affective and stressrelated disorders in the Danish workforce. Scand J Work Environ Heal 31:343-351. doi: 10.5271/sjweh.917

Yuh J, Choi S (2017) Sources of social support, job satisfaction, and quality of life among childcare teachers. Soc Sci J. doi: 10.1016/j.soscij.2017.08.002

Zavidovique L, Gilbert F, Vercambre-Jacquot M-N (2017) Bien-être au travail et qualité de vie des enseignants : quelles différences selon l'ancienneté ? Arch des Mal Prof l'Environnement 1-15. doi: 10.1016/j.admp.2017.09.005 


\section{TABLES \& FIGURES}

Table 1 Population characteristics by level of social support at work

\begin{tabular}{|c|c|c|c|c|c|}
\hline & \multicolumn{5}{|c|}{ Social support at work ${ }^{1}$} \\
\hline & All & Low & Medium & High & $\mathrm{p}^{4}$ \\
\hline \multirow{3}{*}{$\begin{array}{l}n \\
\text { Score of social support at work }{ }^{1} \text {, } \\
m \pm S D \\
\text { [range] }\end{array}$} & 2,473 & 728 & 722 & 1,023 & \\
\hline & $22.5 \pm 3.8$ & $18.0 \pm 2.2$ & $22.1 \pm 0.8$ & $25.9 \pm 2.2$ & \\
\hline & [8-32] & {$[8-20]$} & [21-23] & [24-32] & \\
\hline \multicolumn{6}{|l|}{ Sociodemographic characteristics } \\
\hline Age (years), $\mathrm{m} \pm \mathrm{SD}$ & $43.9 \pm 9.7$ & $45.1 \pm 9.6$ & $43.6 \pm 9.7$ & $43.2 \pm 9.8$ & $<0.01$ \\
\hline \multicolumn{6}{|l|}{ Sex $(\%)$} \\
\hline Female & 66.8 & 70.0 & 65.3 & 65.6 & 0.12 \\
\hline Male & 33.2 & 30.0 & 34.7 & 34.4 & \\
\hline \multicolumn{6}{|l|}{ Work-related characteristics } \\
\hline \multicolumn{6}{|l|}{ Teaching level (\%) } \\
\hline Nursery school & 11.5 & 12.1 & 11.8 & 10.8 & $<0.01$ \\
\hline Primary & 23.9 & 27.7 & 22.9 & 22.0 & \\
\hline Secondary - Middle school & 28.1 & 19.4 & 30.2 & 32.8 & \\
\hline Secondary - High school & 16.3 & 18.0 & 15.5 & 15.8 & \\
\hline Secondary - Vocational high school & 9.8 & 10.4 & 9.0 & 9.9 & \\
\hline University & 10.4 & 12.4 & 10.7 & 8.8 & \\
\hline \multicolumn{6}{|l|}{ Type of school (\%) } \\
\hline Public & 77.0 & 79.4 & 80.5 & 73.0 & 0.01 \\
\hline Public in priority education area & 11.7 & 12.5 & 9.6 & 12.5 & \\
\hline Private (under contract) & 11.3 & 8.1 & 9.9 & 14.5 & \\
\hline \multicolumn{6}{|l|}{ School size (\%) } \\
\hline$\leq 199$ & 25.8 & 26.4 & 26.0 & 25.3 & 0.01 \\
\hline 200-999 & 54.1 & 50.1 & 53.3 & 57.4 & \\
\hline$\geq 1000$ & 20.1 & 23.5 & 20.7 & 17.3 & \\
\hline \multicolumn{6}{|l|}{ School location (\%) } \\
\hline Rural & 22.8 & 20.4 & 21.7 & 24.0 & 0.16 \\
\hline Suburban & 47.7 & 51.7 & 46.7 & 47.4 & \\
\hline Urban & 29.6 & 27.9 & 31.6 & 28.6 & \\
\hline \multicolumn{6}{|l|}{ Psychosocial work environment } \\
\hline Score of psychological demand ${ }^{1}, m \pm S D$ & $23.9 \pm 4.1$ & $24.8 \pm 4.2$ & $23.8 \pm 4.0$ & $23.2 \pm 3.9$ & $<0.01$ \\
\hline Score of decision latitude ${ }^{1}, m \pm S D$ & $76.6 \pm 8.3$ & $74.4 \pm 9.1$ & $76.0 \pm 7.9$ & $78.6 \pm 7.6$ & $<0.01$ \\
\hline Psychological violence² (\%) & & & & & $<0.01$ \\
\hline Neither victim nor witness & 52.6 & 42.5 & 51.3 & 60.5 & \\
\hline Witness & 30.2 & 27.4 & 33.0 & 30.3 & \\
\hline Victim & 17.2 & 30.1 & 15.7 & 9.2 & \\
\hline \multicolumn{6}{|l|}{ Private social \& environmental context } \\
\hline \multicolumn{6}{|l|}{ Marital status (\%) } \\
\hline Single & 21.5 & 19.7 & 24.6 & 20.6 & 0.03 \\
\hline Married, cohabitation & 66.1 & 65.6 & 63.5 & 68.3 & \\
\hline Separated, divorced, widowed & 12.4 & 14.7 & 11.9 & 11.1 & \\
\hline With young child at home (<7 years) $(\%)$ & 26.4 & 23.9 & 25.6 & 28.8 & 0.07 \\
\hline Social relationships scale ${ }^{3}, m \pm S D$ & $11.5 \pm 2.1$ & $11.0 \pm 2.2$ & $11.4 \pm 2.0$ & $12.0 \pm 1.9$ & $<0.01$ \\
\hline Environment scale ${ }^{3}, \mathrm{~m} \pm \mathrm{SD}$ & $30.0 \pm 4.5$ & $28.6 \pm 4.9$ & $30.1 \pm 3.9$ & $30.8 \pm 4.4$ & $<0.01$ \\
\hline
\end{tabular}

$\mathrm{m} \pm$ SD: mean \pm standard deviation; ${ }^{1}$ Derived from the Job Content Questionnaire (Niedhammer et al., 2002); ${ }^{2}$ Self-reported exposure based on the definition of Leymann's psychological violence questionnaire (Niedhammer et al., 2006); ${ }^{3}$ Derived from the WHOQOL-BREF questionnaire (Baumann et al., 2010); ${ }^{4}$ Chi-squared tests for categorical variables and t-tests for continuous variables. 
Table 2 Pearson's correlation coefficients between scores of social support at work, private social and environmental context and the three dimensions of burnout

\begin{tabular}{|c|c|c|c|c|c|c|c|c|c|c|c|}
\hline & & 1 & 2 & 3 & 4 & 5 & 6 & 7 & 8 & 9 & 10 \\
\hline \multirow{5}{*}{ Social support at work ${ }^{1}$} & 1. Global & 1.00 & & & & & & & & & \\
\hline & 2. From coworkers & 0.71 & 1.00 & & & & & & & & \\
\hline & 3. From supervisors & 0.83 & 0.20 & 1.00 & & & & & & & \\
\hline & 4. Instrumental & 0.94 & 0.69 & 0.77 & 1.00 & & & & & & \\
\hline & 5. Emotional & 0.95 & 0.65 & 0.81 & 0.78 & 1.00 & & & & & \\
\hline \multirow{2}{*}{$\begin{array}{l}\text { Private social and } \\
\text { environmental context }{ }^{2}\end{array}$} & 6. Social relationships & 0.24 & 0.22 & 0.16 & 0.23 & 0.22 & 1.00 & & & & \\
\hline & 7. Environment & 0.23 & 0.20 & 0.17 & 0.21 & 0.23 & 0.35 & 1.00 & & & \\
\hline \multirow{3}{*}{ Burnout dimensions ${ }^{3}$} & 8. Emotional exhaustion & -0.22 & 0.13 & -0.20 & 0.20 & -0.21 & -0.25 & -0.37 & 1.00 & & \\
\hline & 9. Depersonalization & -0.15 & -0.12 & -0.11 & -0.13 & -0.14 & -0.18 & -0.18 & 0.41 & 1.00 & \\
\hline & 10. Personal accomplishment & 0.23 & 0.19 & 0.18 & 0.22 & 0.23 & 0.26 & 0.18 & -0.18 & -0.24 & 1.00 \\
\hline
\end{tabular}

4 Pearson's correlation coefficient ; all p-values $<0.0001$

${ }^{1}$ Karasek Job Content Questionnaire (Niedhammer et al., 2002) ;

2 French abbreviated version of the WHOQOL questionnaire (Baumann et al., 2010) ;

${ }^{3}$ Maslach Burnout Inventory (Maslach \& Jackson, 1981). 
Table 3 Association between social support at work and burnout before and after adjustment for private context factors

\begin{tabular}{|c|c|c|c|c|}
\hline \multirow[b]{3}{*}{$n(\%)$} & \multicolumn{4}{|c|}{$\begin{array}{c}\text { Social support at work }{ }^{1} \\
\text { OR }[95 \% \mathrm{Cl}]\end{array}$} \\
\hline & Low (ref.) & Medium & High & $\begin{array}{c}p \text {-value } \\
\text { for trend }\end{array}$ \\
\hline & $728(29.4)$ & $722(29.2)$ & $1023(41.4)$ & \\
\hline Burnout $(1+2+3), \%$ cases $^{2}$ & $12.1 \%$ & $8.2 \%$ & $3.5 \%$ & \\
\hline Model 1 & 1 & $0.64[0.45,0.91]$ & $0.27[0.18,0.41]$ & $<0.01$ \\
\hline Model 2 & 1 & $0.91[0.62,1.32]$ & $0.52[0.33,0.80]$ & $<0.01$ \\
\hline Model 3 & 1 & $1.08[0.73,1.60]$ & $0.62[0.40,0.98]$ & 0.05 \\
\hline \multicolumn{5}{|c|}{ 1) High emotional exhaustion ${ }^{2}$} \\
\hline Model 1 & 1 & $0.67[0.54,0.84]$ & $0.44[0.36,0.55]$ & $<0.01$ \\
\hline Model 2 & 1 & $0.93[0.73,1.20]$ & $0.75[0.58,0.95]$ & 0.02 \\
\hline Model 3 & 1 & $1.04[0.80,1.34]$ & $0.86[0.67,1.11]$ & 0.22 \\
\hline \multicolumn{5}{|c|}{ 2) High depersonalization ${ }^{2}$} \\
\hline Model 1 & 1 & $0.69[0.55,0.87]$ & $0.58[0.47,0.72]$ & $<0.01$ \\
\hline Model 2 & 1 & $0.86[0.67,1.10]$ & $0.85[0.67,1.08]$ & 0.18 \\
\hline Model 3 & 1 & $0.89[0.70,1.15]$ & $0.92[0.72,1.18]$ & 0.54 \\
\hline \multicolumn{5}{|c|}{ 3) Reduced personal accomplishment 2} \\
\hline Model 1 & 1 & $0.81[0.65,1.00]$ & $0.43[0.35,0.53]$ & $<0.01$ \\
\hline Model 2 & 1 & $0.87[0.69,1.09]$ & $0.52[0.41,0.65]$ & $<0.01$ \\
\hline Model 3 & 1 & $0.94[0.74,1.18]$ & $0.59[0.47,0.75]$ & $<0.01$ \\
\hline
\end{tabular}

Odds ratio $(\mathrm{OR})$ and $95 \%$ confidence interval $[95 \% \mathrm{Cl}]$ from logistic regression models.

Model 1: adjusted for age and sex.

Model 2: Model 1 + work-related characteristics (i.e., teaching level, type of school, school size, zscores of decision latitude and psychological demand, and psychological violence).

Model 3: Model 2 + teachers' private social and environmental context (i.e., z-scores of "social relationships" and "environment" subscales of the WHOQOL-BREF).

${ }^{1}$ Social support at work was derived from the Job Content Questionnaire (Niedhammer et al., 2002) and was categorized in tertiles.

2 The burnout dimensions were dichotomized using extreme tertiles. Burnout cases were defined as those with a high score ( $3^{\text {rd }}$ tertile) on emotional exhaustion and depersonalization and a low score ( $1^{\text {st }}$ tertile) on personal accomplishment.

${ }^{3}$ The test for a linear trend was computed by considering the level of social support as an ordinal variable. 
Table 4 Association between social support at work (by source and type) and burnout after adjustment for private context factors

\begin{tabular}{|c|c|c|c|c|}
\hline & \multicolumn{4}{|c|}{$\begin{array}{l}\text { Social support at work }{ }^{1} \\
\text { OR }[95 \% \mathrm{Cl}]\end{array}$} \\
\hline & Low (ref.) & Medium & High & $\begin{array}{c}p \text {-value } \\
\text { for trend }\end{array}$ \\
\hline \multicolumn{5}{|l|}{ Burnout $(1+2+3)^{2}$} \\
\hline From supervisors & 1 & $0.84[0.58,1.21]$ & $0.43[0.27,0.71]$ & $<0.01$ \\
\hline From coworkers & 1 & $0.63[0.42,0.95]$ & $0.79[0.53,1.18]$ & 0.20 \\
\hline Emotional & 1 & $0.62[0.37,1.01]$ & $0.50[0.34,0.73]$ & $<0.01$ \\
\hline Instrumental & 1 & $1.06[0.71,1.59]$ & $0.77[0.49,1.21]$ & 0.23 \\
\hline \multicolumn{5}{|c|}{ 1) High emotional exhaustion ${ }^{2}$} \\
\hline From supervisors & 1 & $0.86[0.68,1.11]$ & $0.85[0.66,1.11]$ & 0.25 \\
\hline From coworkers & 1 & $0.68[0.53,0.87]$ & $0.87[0.67,1.13]$ & 0.36 \\
\hline Emotional & 1 & $0.83[0.61,1.14]$ & $0.80[0.64,1.01]$ & 0.07 \\
\hline Instrumental & 1 & $0.95[0.72,1.25]$ & $0.79[0.59,1.05]$ & 0.07 \\
\hline \multicolumn{5}{|c|}{ 2) High depersonalization ${ }^{2}$} \\
\hline From supervisors & 1 & $0.94[0.74,1.19]$ & $0.91[0.71,1.17]$ & 0.48 \\
\hline From coworkers & 1 & $0.83[0.65,1.05]$ & $0.90[0.70,1.15]$ & 0.43 \\
\hline Emotional & 1 & $0.77[0.57,1.04]$ & $0.78[0.62,0.97]$ & 0.03 \\
\hline Instrumental & 1 & $0.96[0.74,1.25]$ & $1.01[0.78,1.32]$ & 0.84 \\
\hline \multicolumn{5}{|c|}{ 3) Reduced personal accomplishment ${ }^{2}$} \\
\hline From supervisors & 1 & $0.90[0.71,1.12]$ & $0.56[0.44,0.72]$ & $<0.01$ \\
\hline From coworkers & 1 & $0.73[0.58,0.92]$ & $0.67[0.53,0.86]$ & $<0.01$ \\
\hline Emotional & 1 & $0.82[0.62,1.09]$ & $0.68[0.55,0.85]$ & $<0.01$ \\
\hline Instrumental & 1 & $0.98[0.77,1.26]$ & $0.67[0.52,0.87]$ & $<0.01$ \\
\hline
\end{tabular}

Odds ratio and $95 \%$ confidence interval from logistic regression models adjusted for age, sex, workrelated characteristics (i.e., teaching level, type of school, school size, z-scores of decision latitude and psychological demand, and psychological violence) and teachers' private social and environmental context (i.e., z-scores of "social relationships" and "environment" subscales of the WHOQOL-BREF). The model is mutually adjusted for social support from coworkers and from supervisors, respectively, on instrumental and emotional support at work.

${ }^{1}$ Social support at work was derived from the Job Content Questionnaire (Niedhammer et al., 2002) and was categorized in tertiles.

2 The burnout dimensions were dichotomized using extreme tertiles. Burnout cases were defined as those with a high score ( $3^{\text {rd }}$ tertile) on emotional exhaustion and depersonalization and a low score $\left(1^{\text {st }}\right.$ tertile) on personal accomplishment.

3 The test for a linear trend was computed by considering the level of social support as an ordinal variable. 